Research Article

\title{
Nephroprotective Effect of Mesenchymal Stem Cell-Based Therapy of Kidney Disease Induced by Toxicants
}

\author{
Shujun Lin, Wenshan Lin, Chunling Liao, and Tianbiao Zhou \\ Department of Nephrology, The Second Affiliated Hospital, Shantou University Medical College, 515041 Shantou, China \\ Correspondence should be addressed to Tianbiao Zhou; zhoutb@aliyun.com
}

Received 27 August 2020; Revised 28 October 2020; Accepted 10 December 2020; Published 23 December 2020

Academic Editor: Erika B. Rangel

Copyright (C) 2020 Shujun Lin et al. This is an open access article distributed under the Creative Commons Attribution License, which permits unrestricted use, distribution, and reproduction in any medium, provided the original work is properly cited.

\begin{abstract}
Background. Renal damage caused by drug toxicity is becoming increasingly common in the clinic. Preventing and treating kidney damage caused by drug toxicity are essential to maintain patient health and reduce the social and economic burden. In this study, we performed a meta-analysis to assess the nephroprotective effect of mesenchymal stem cells (MSCs) in the treatment of kidney disease induced by toxicants. Methods. The Cochrane Library, Embase, ISI Web of Science, and PubMed databases were searched up to December 31,2019, to identify studies and extract data to assess the efficacy of MSCs treatment of kidney disease induced by toxicants using Cochrane Review Manager Version 5.3. A total of 27 studies were eligible and selected for this meta-analysis. Results. The results showed that a difference in serum creatinine levels between the MSC treatment group and control group was observed for $2,4,5,6-8,10-15,28-30$, and $\geq 42$ days ( 2 days: $\mathrm{WMD}=-0.88,95 \%$ CI: $-1.34,-0.42, P=0.0002 ; 4$ days: WMD $=-$ $0.74,95 \%$ CI: $-0.95,-0.54, P<0.00001 ; 5$ days: $\mathrm{WMD}=-0.46,95 \% \mathrm{CI}:-0.67,-0.25, P<0.0001 ; 6-8$ days: $\mathrm{WMD}=-0.55,95 \%$ CI: $-0.84,-0.26, P=0.0002 ; 10-15$ days: $\mathrm{WMD}=-0.37,95 \% \mathrm{CI}:-0.53,-0.20, P<0.0001 ; 28-30$ days: $\mathrm{WMD}=-0.53,95 \% \mathrm{CI}$ : $-1.04,-0.02, P=0.04 ; \geq 42$ days: $\mathrm{WMD}=-0.22,95 \% \mathrm{CI}:-0.39,-0.06, P=0.007)$. Furthermore, a difference in blood urea nitrogen levels between the MSC treatment group and control group was observed for 2-3, 4-5, 6-8, and $\geq 28$ days. The results also indicate that MSC treatment alleviated inflammatory cells, necrotic tubules, regenerative tubules, and renal interstitial fibrosis in kidney disease induced by toxicants. Conclusion. MSCs may be a promising therapeutic agent for kidney disease induced by toxicants.
\end{abstract}

\section{Introduction}

Kidney injury occurs during acute kidney injury (AKI) and chronic kidney disease (CKD), and it is a common condition associated with the morbidity and mortality of patients. A total of $80 \%$ of patients who survive an AKI episode completely recover kidney function, and recovered AKI patients present an almost 9-fold increase in risk for CKD development [1]. Toxicant-induced kidney injury is one of the most common causes of kidney disease, causing substantial morbidity and hampering drug development [2]. At present, renal damage caused by drug toxicity is becoming increasingly common in the clinic. Preventing and treating kidney damage caused by drug toxicity is essential to maintain patient health and reduce the social and economic burden.
Mesenchymal stem cells (MSCs), which are multipotent mesenchymal cells present in various tissues, have multilineage differentiation ability under appropriate conditions and are easy to obtain. They are a promising therapeutic option for some diseases because of their unique property of releasing some important bioactive factors [3-5]. Drug toxicity can induce renal tubular epithelial cell damage or death and can lead to renal interstitial inflammation, which eventually develops into renal interstitial fibrosis and renal loss. Previous studies have shown that MSCs can play a protective role against injury of renal tubular epithelial cells and prevent renal interstitial fibrosis [6-10]. Before clinical application, animal experiments in vivo are generally required to confirm the effectiveness of MSCs. Furthermore, there are few clinical trials of MSCs on kidney disease induced by toxicants. Therefore, in this study, we performed a meta-analysis to assess the 


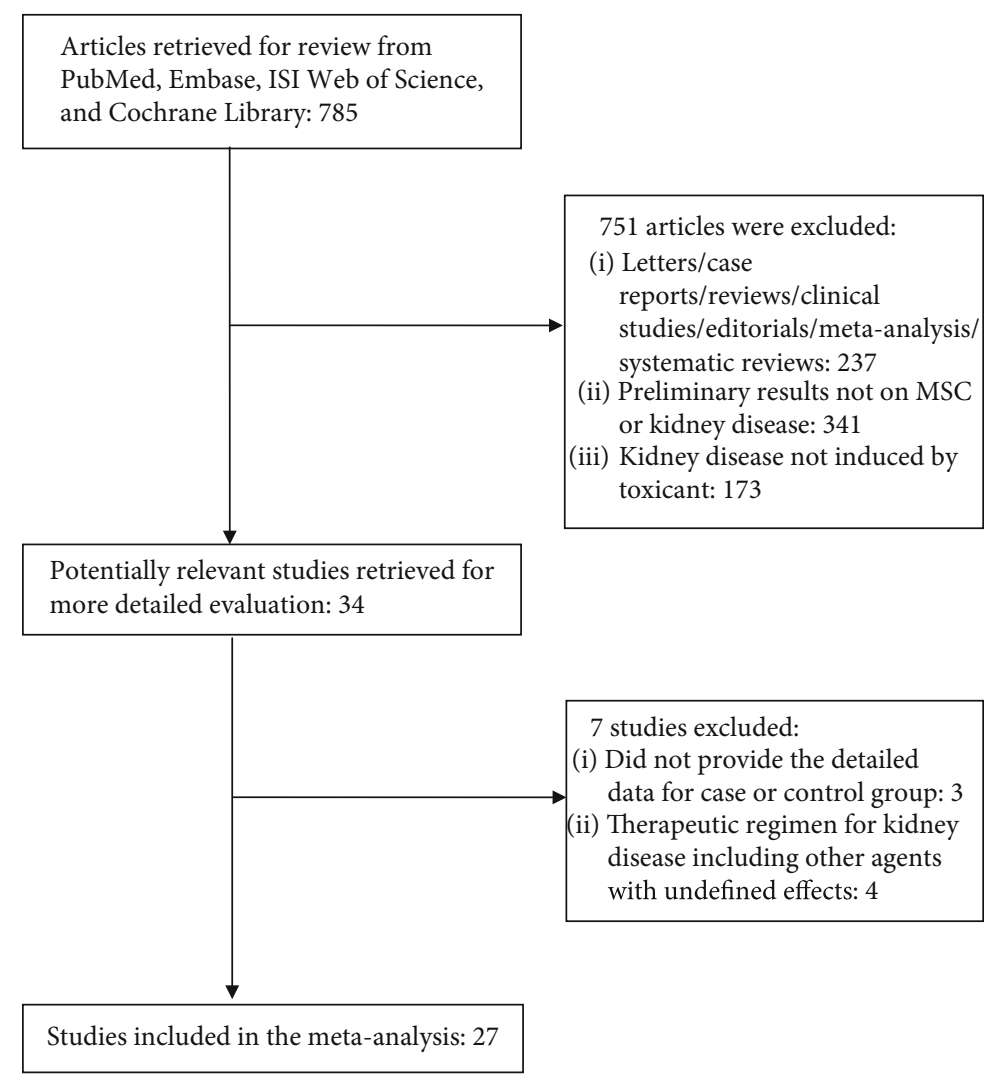

Figure 1: Flow diagram of the selection process.

nephroprotective effect of MSCs in the treatment of kidney disease induced by toxicants in animals.

\section{Materials and Methods}

2.1. Search Strategy. We searched databases (Cochrane Library, Embase, ISI Web of Science, and PubMed) up to Dec 31, 2019, using the following search terms: (mesenchymal stem cells OR MSC OR MSCs OR multipotent stromal cells OR mesenchymal stromal cells OR mesenchymal progenitor cells OR stem cells) AND (gentamicin OR aristolochic acid OR cisplatin OR adriamycin OR cadmium chloride OR methotrexate OR rifampicin OR glycerol OR streptozocin) AND (kidney injury OR renal failure OR kidney disease). The search was confined to English-language literature. An additional search was conducted among the eligible manual references of the cited articles.

2.2. Inclusion and Exclusion Criteria. Our meta-analysis included studies analyzing the efficacy of MSC treatment in mice or rats with kidney disease. The following studies were excluded from the analysis: (1) letters, case reports, reviews, clinical studies, editorials, meta-analysis, and systematic reviews; (2) studies lacking the targeted indicators or number of case or control groups and were conducted in humans; (3) studies of kidney disease that was not induced by toxicants; and (4) studies with therapeutic regimen for kidney disease that included other agents with undefined effects.
2.3. Outcome Measures. We filtered the following outcomes associated with the efficacy of MSC treatment from the recruited studies: serum creatinine (Scr), blood urea nitrogen (BUN), urinary albumin excretion (UAE), malondialdehyde (MDA), L-glutathione (GSH), superoxide dismutase (SOD), and renal pathology. In addition, we conducted a mutual consensus when met with disagreements.

2.4. Quality Assessment. Two investigators independently evaluated the methodological quality using the Cochrane Handbook for Interventions. We assessed the following sections of every investigation: selection bias, attrition bias, performance bias, detection bias, reporting bias, and other bias. Each item was classified as unclear, high risk, or low risk.

2.5. Statistical Analysis. Review Manager Version 5.3 was applied to explore whether MSC treatment achieved a good efficacy in kidney disease induced by toxicants, and STATA 12.0 was used to test the publication bias. Heterogeneity of variation among individual studies was quantified and described using $I^{2}$. The fixed effects model was used if the $P$ value of the heterogeneity test was $\geq 0.1$. Otherwise, the random effects model was applied to pool the outcomes. In addition, to compute continuous variables, we analyzed weighted mean differences (WMDs) for the mean values. We also calculated $95 \%$ confidence intervals $(95 \% \mathrm{CI})$ for the included studies using the Mantel-Haenszel (M-H) method. Additionally, we evaluated the publication bias using Begg's rank 


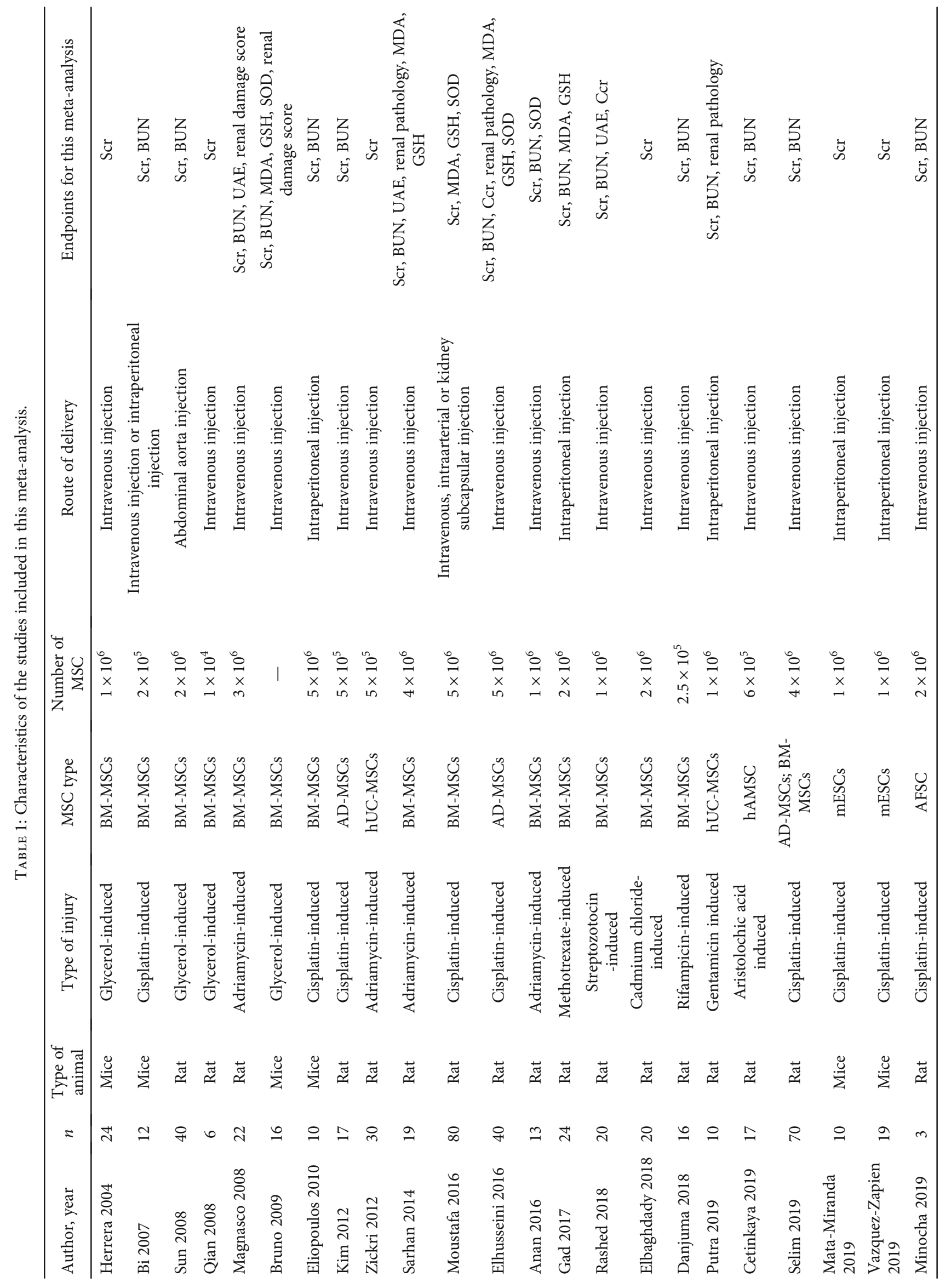




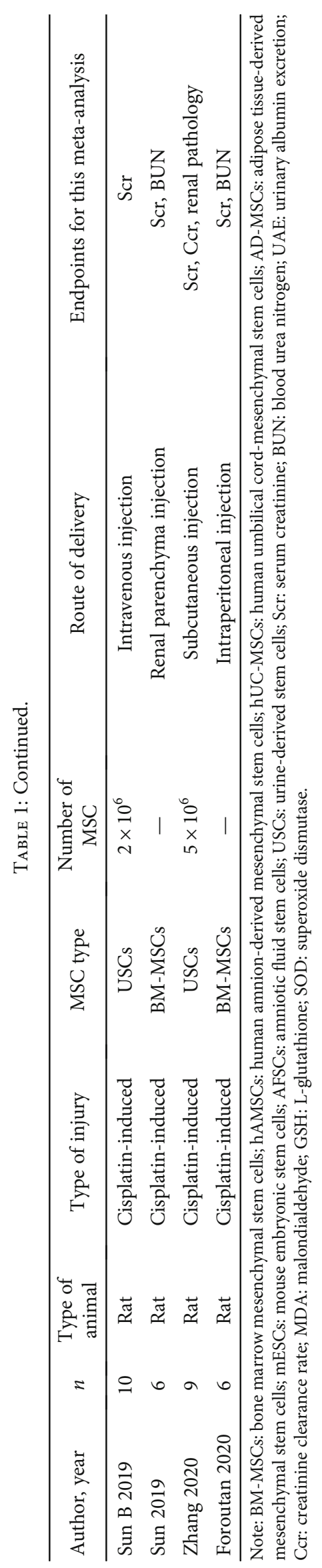




\begin{tabular}{|c|c|c|c|c|c|c|c|}
\hline & 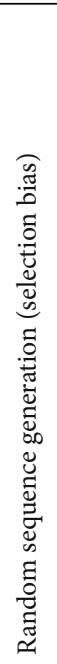 & 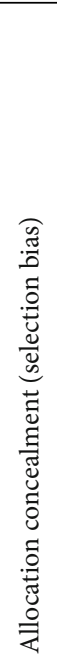 & 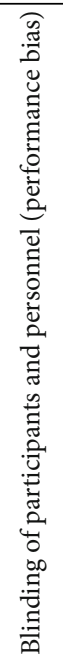 & 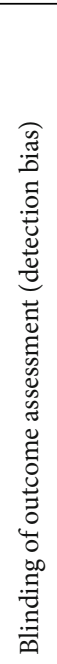 & 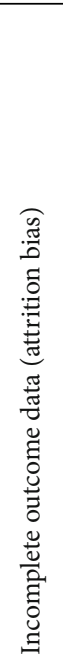 & 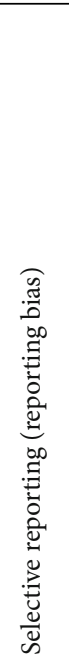 & 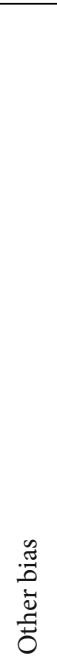 \\
\hline Anan 2016 & $?$ & $?$ & $?$ & $?$ & + & + & + \\
\hline Bi 2007 & $?$ & $?$ & $?$ & + & + & + & + \\
\hline Bruno 2009 & $?$ & $?$ & $?$ & + & + & + & + \\
\hline Cetinkaya 2019 & + & $?$ & $?$ & $?$ & + & + & + \\
\hline Danjuma 2018 & $?$ & $?$ & $?$ & $?$ & + & + & + \\
\hline Elbaghdady 2018 & + & $?$ & $?$ & $?$ & + & + & + \\
\hline Elhusseini 2016 & $?$ & $?$ & $?$ & $?$ & + & + & + \\
\hline Eliopoulos 2010 & $?$ & $?$ & $?$ & $?$ & + & + & + \\
\hline Foroutan 2020 & $?$ & $?$ & $?$ & $?$ & + & + & + \\
\hline Gad 2017 & $?$ & $?$ & $?$ & $?$ & + & + & + \\
\hline Herrera 2004 & $?$ & $?$ & $?$ & $?$ & + & + & + \\
\hline Kim 2012 & $?$ & $?$ & $?$ & + & + & + & + \\
\hline Magnasco 2008 & $?$ & $?$ & $?$ & $?$ & + & + & + \\
\hline Mata-Miranda 2019 & + & $?$ & $?$ & $?$ & + & + & + \\
\hline Minocha 2019 & + & $?$ & $?$ & + & + & + & + \\
\hline Moustafa 2016 & $?$ & $?$ & $?$ & $?$ & + & + & + \\
\hline Putra 2019 & + & $?$ & $?$ & $?$ & + & + & + \\
\hline Qian 2008 & $?$ & $?$ & $?$ & $?$ & + & + & + \\
\hline Rashed 2018 & + & $?$ & $?$ & $?$ & + & + & + \\
\hline Sarhan 2014 & $?$ & $?$ & $?$ & + & + & + & + \\
\hline Selim 2019 & $?$ & $?$ & $?$ & $?$ & + & + & + \\
\hline Sun 2008 & + & $?$ & $?$ & + & + & + & + \\
\hline Sun 2019 & $?$ & $?$ & $?$ & + & + & + & + \\
\hline Sun B 2019 & + & $?$ & $?$ & + & + & + & + \\
\hline Vazquez-Zapien 2019 & + & $?$ & $?$ & $?$ & + & + & + \\
\hline Zhang 2020 & $?$ & $?$ & $?$ & $?$ & + & + & + \\
\hline Zickri 2012 & $?$ & $?$ & $?$ & $?$ & + & + & + \\
\hline
\end{tabular}

(a)

FIGURE 2: Continued. 


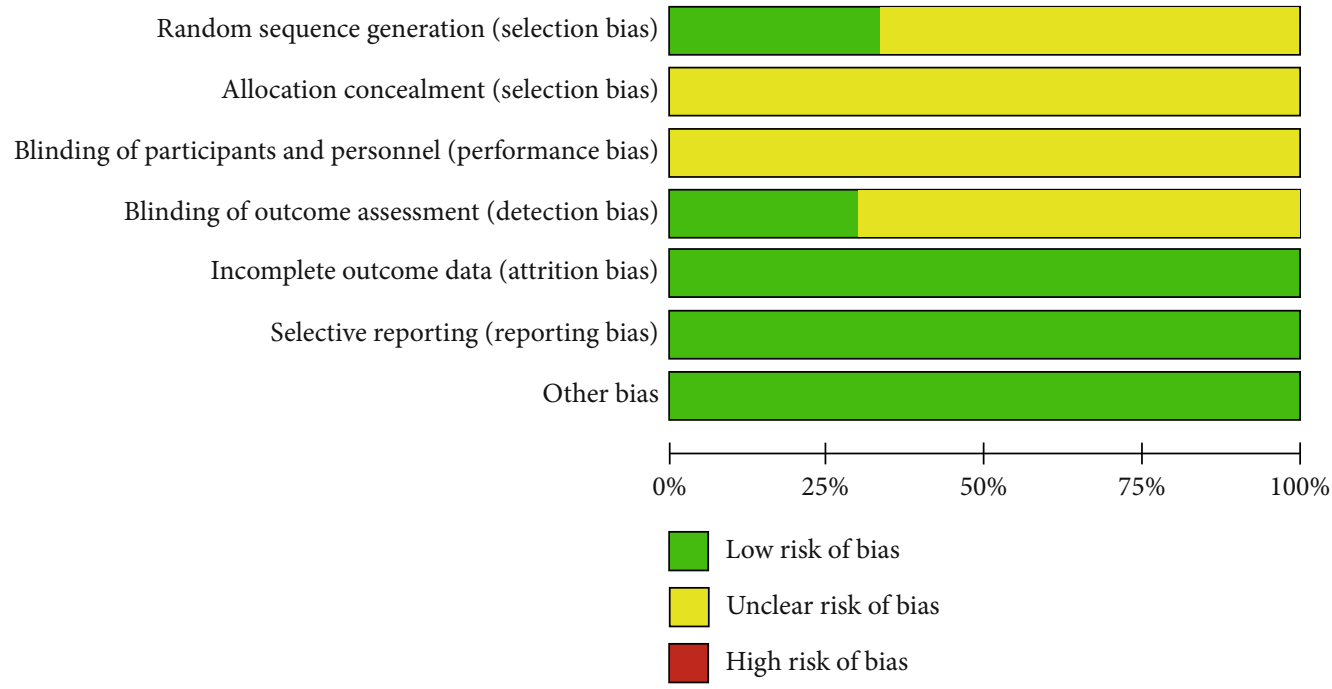

(b)

Figure 2: (a) Aggregate Risk of bias graph for each experimental animal studies; “?”: Unclear risk; “+”: Low risk. (b) Risk of bias summary.

correlation test as well as Egger's linear regression method among the studies. A $P$ value $<0.05$ was considered of statistical significance.

\section{Results}

3.1. Search Results. The databases mentioned above were searched, and only studies in mice or rats that evaluated the therapeutic efficacy of MSC treatment on kidney disease induced by toxicants were selected. Twenty-seven studies [11-37] were eligible and selected for this meta-analysis, and a flowchart of inclusion of studies is presented in Figure 1. Study characteristics are shown in Table 1.

3.2. Quality Assessment of Included Studies. The methodological quality of the selected studies was considered acceptable because most study domains were ranked as unclear risk or low risk of bias. Unclear risk of bias was mostly detected in performance and selection bias. Low risk of bias mostly occurred in detection, reporting, and attrition bias. Figure 2 shows a summary of the risk of biases of the selected studies.

3.3. Scr. A total of 27 studies [11-37] were selected to assess the effect of MSCs on Scr, and the results show that a difference between the MSC treatment and control groups was observed for $2,4,5,6-8,10-15,28-30$ days, and $\geq 42$ days ( 2 days: $\mathrm{WMD}=-0.88,95 \% \mathrm{CI}:-1.34,-0.42, P=0.0002 ; 4$ days: $\mathrm{WMD}=-0.74,95 \% \mathrm{CI}:-0.95,-0.54, P<0.00001 ; 5$ days: $\mathrm{WMD}=-0.46,95 \% \mathrm{CI}:-0.67,-0.25, P<0.0001 ; 6-8$ days: $\mathrm{WMD}=-0.55,95 \% \mathrm{CI}:-0.84,-0.26, P=0.0002 ; 10-15$ days: $\mathrm{WMD}=-0.37,95 \% \mathrm{CI}:-0.53,-0.20, P<0.0001 ; 28-30$ days: $\mathrm{WMD}=-0.53,95 \% \mathrm{CI}:-1.04,-0.02, P=0.04 ; \geq 42$ days: $\mathrm{WMD}=-0.22,95 \% \mathrm{CI}:-0.39,-0.06, P=0.007$; Figure 3 and Table 2). However, no difference was observed between the MSC treatment and control groups for 3 days (3 days:
$\mathrm{WMD}=-0.09,95 \% \mathrm{CI}:-0.25,-0.06, P=0.24$; Figure 3 and Table 2).

3.4. BUN. A total of 18 studies $[11-15,17-19,21,22,24,26-$ $29,32-34,36,37]$ were selected to assess the effect of MSCs on BUN, and the results indicate that the difference between the MSC treatment and control groups was observed for 2-3, $4-5,6-8$, and $\geq 28$ days ( $2-3$ days: $\mathrm{WMD}=-25.08,95 \% \mathrm{CI}$ : $-37.49,-12.67, P<0.0001 ; 4-5$ days: $\mathrm{WMD}=-45.71,95 \%$ CI: $-59.36,-32.05, P<0.00001 ; 6-8$ days: $\mathrm{WMD}=-57.55$, 95\% CI: $-99.19,-15.91, P=0.007 ; \geq 28$ days: $\mathrm{WMD}=-23.39$ , 95\% CI: $-36.39,-10.40, P=0.0004$; Figure 4 and Table 2). However, no difference was observed between the MSC treatment and control groups for 13-15 days (WMD $=-13.40$, 95\% CI: $-32.34,5.54, P=0.17$; Figure 4 and Table 2).

3.5. Urinary Albumin Excretion. Three studies [22, 26, 27] were selected in the meta-analysis for the assessment of MSCs on UAE. The results show that the MSC group had a lower UAE than the control group (WMD $=-22.66,95 \%$ CI: $-26.41,-18.90, P<0.00001$; Table 2).

3.6. Oxidative Stress. Four studies [17, 19, 23, 27] were selected for the assessment of MDA, four [17, 19, 23, 27] for GSH, and three $[11,17,23]$ for SOD. The results indicate that a difference between the MSC treatment and control groups was observed for MDA, GSH, and SOD (MDA: WMD $=-17.21,95 \% \mathrm{CI}:-20.38,-14.04, P<0.00001 ; \mathrm{GSH}$ : $\mathrm{WMD}=4.62,95 \% \mathrm{CI}: 2.74,6.50, P<0.00001$; SOD: WMD $=5.42$, 95\% CI: 2.92, 7.93, $P<0.0001$; Table 2 ).

3.7. Assessment of Renal Pathology. Four studies [17, 24, 27, 35] for inflammatory cells, two studies [17, 27] for necrotic tubules, two studies $[17,27]$ for regenerative tubules, and three studies $[17,27,35]$ for renal interstitial fibrosis were included in this meta-analysis. The results indicate that the difference in inflammatory cells, necrotic tubules, 


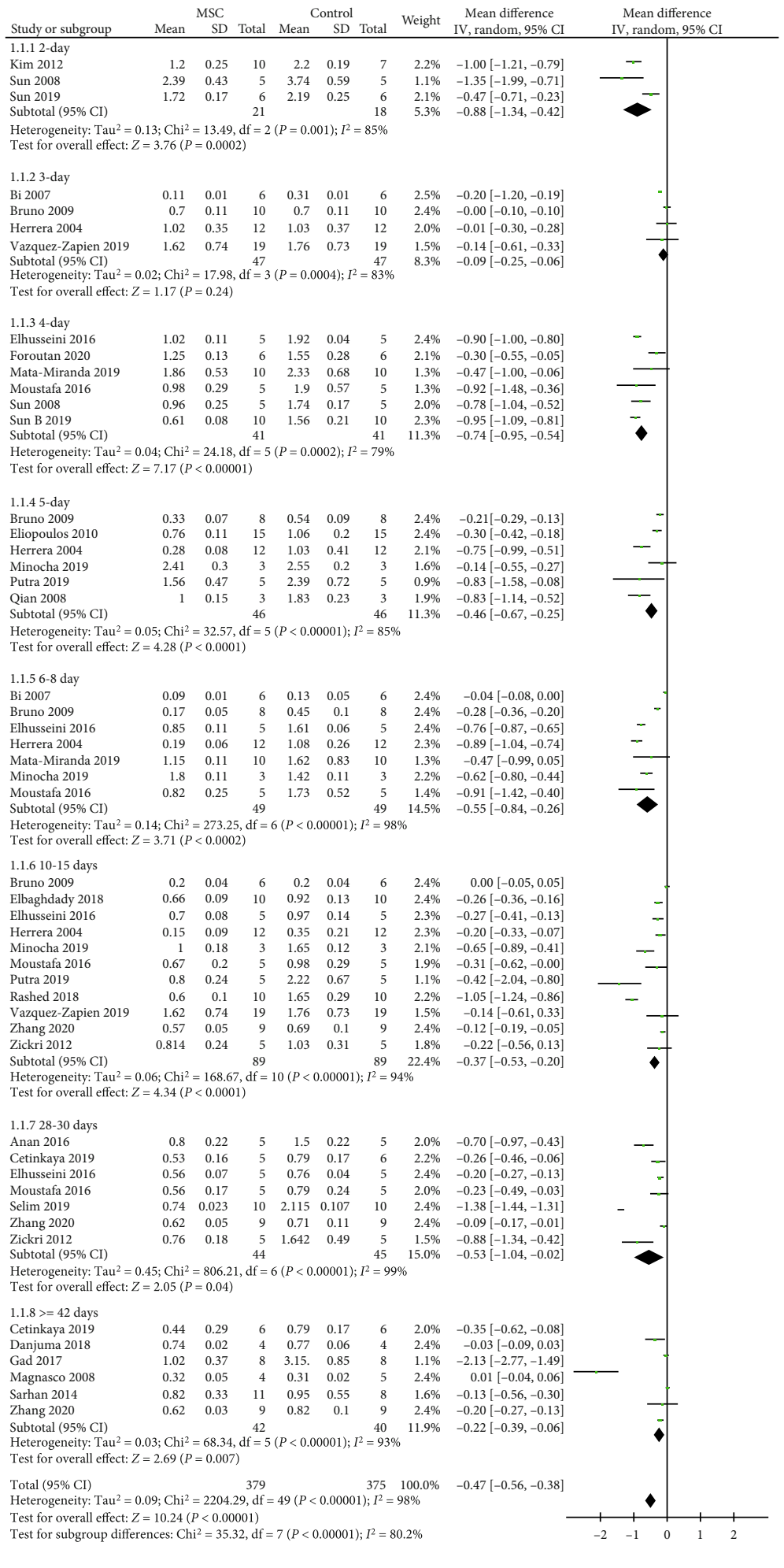

Favours MSC Favours control

FIgURE 3: Effect of MSC on Scr. 
TABLE 2: Meta-analysis of the efficacy of MSC in therapy of renal injury induced by toxicant.

\begin{tabular}{|c|c|c|c|c|c|c|}
\hline Indicators & Time point & $\begin{array}{l}\text { Studies } \\
\text { number }\end{array}$ & $\begin{array}{c}Q \text { test } \\
P \text { value }\end{array}$ & $\begin{array}{c}\text { Model } \\
\text { selected }\end{array}$ & $\begin{array}{c}\text { OR/WMD } \\
(95 \% \mathrm{CI})\end{array}$ & $P$ \\
\hline \multirow{8}{*}{ Scr } & 2 days & 3 & 0.001 & Random & $-0.88(-1.34,-0.42)$ & 0.0002 \\
\hline & 3 days & 4 & 0.0004 & Random & $-0.09(-0.25,0.06)$ & 0.24 \\
\hline & 4 days & 6 & 0.0002 & Random & $-0.74(-0.95,-0.54)$ & $<0.00001$ \\
\hline & 5 days & 6 & $<0.00001$ & Random & $-0.46(-0.67,-0.25)$ & $<0.0001$ \\
\hline & 6-8 days & 7 & $<0.00001$ & Random & $-0.55(-0.84,-0.26)$ & 0.0002 \\
\hline & 10-15 days & 11 & $<0.00001$ & Random & $-0.37(-0.53,-0.20)$ & $<0.0001$ \\
\hline & 28-30 days & 7 & $<0.00001$ & Random & $-0.53(-1.04,-0.02)$ & 0.04 \\
\hline & $\geq 42$ days & 6 & $<0.00001$ & Random & $-0.22(-0.39,-0.06)$ & 0.007 \\
\hline \multirow{5}{*}{ BUN } & 2-3 days & 6 & $<0.00001$ & Random & $-25.08(-37.49,-12.67)$ & $<0.0001$ \\
\hline & 4-5 days & 8 & $<0.00001$ & Random & $-45.71(-59.36,-32.05)$ & $<0.00001$ \\
\hline & 6-8 days & 5 & $<0.00001$ & Random & $-57.55(-99.19,-15.91)$ & 0.007 \\
\hline & 13-15 days & 4 & $<0.00001$ & Random & $-13.40(-32.34,5.54)$ & 0.17 \\
\hline & $\geq 28$ days & 8 & $<0.00001$ & Random & $-23.39(-36.39,-10.40)$ & 0.0004 \\
\hline UAE & - & 3 & 0.72 & Fixed & $-22.66(-26.41,-18.90)$ & $<0.00001$ \\
\hline MDA & - & 4 & 0.41 & Fixed & $-17.21(-20.38,-14.04)$ & $<0.00001$ \\
\hline GSH & - & 4 & $<0.00001$ & Random & $4.62(2.74,6.50)$ & $<0.00001$ \\
\hline SOD & - & 3 & $<0.00001$ & Random & $5.42(2.92,7.93)$ & $<0.0001$ \\
\hline \multicolumn{7}{|l|}{ Renal pathology } \\
\hline Inflammatory cells & - & 4 & $<0.00001$ & Random & $-2.66(-3.83,-1.49)$ & $<0.00001$ \\
\hline Necrotic tubule & - & 2 & $<0.00001$ & Random & $-2.58(-4.75,-0.40)$ & 0.02 \\
\hline Regenerative tubules & - & 2 & - & Fixed & $6.00(3.45,8.55)$ & $<0.00001$ \\
\hline Renal interstitial fibrosis & - & 3 & $<0.00001$ & Random & $-5.82(-7.41,-4.23)$ & $<0.00001$ \\
\hline
\end{tabular}

Note: Scr: serum creatinine; BUN: blood urea nitrogen; UAE: urinary albumin excretion; Ccr: creatinine clearance rate; MDA: malondialdehyde; GSH: Lglutathione; SOD: superoxide dismutase.

regenerative tubules, and renal interstitial fibrosis between the MSC treatment and control groups was significant (inflammatory cells: WMD $=-2.66,95 \% \mathrm{CI}:-3.83,-1.49, P$ $<0.00001$; necrotic tubules: WMD $=-2.58,95 \% \mathrm{CI}:-4.75$, $-0.40, P=0.02$; regenerative tubules: $\mathrm{WMD}=6.00,95 \% \mathrm{CI}$ : $3.45,8.55, P<0.00001$; renal interstitial fibrosis: $\mathrm{WMD}=-$ 5.82, 95\% CI: $-7.41,-4.23, P<0.00001$; Table 2 ).

3.8. Publication Bias. Publication bias was tested in this metaanalysis, and a funnel plot generated using STATA 12.0 for the primary outcome. Begg's test and Egger's test results suggest that publication bias was present $(P \leq 0.01$ and $P \leq 0.01$, respectively; Figure 5).

\section{Discussion}

We reviewed all the selected studies and evaluated the Scr, BUN, UAE, oxidative stress, and renal pathology results to assess the nephroprotective effect of MSCs in the treatment of kidney disease induced by toxicants. We found that MSC treatment reduced Scr levels at 2, 4, 5, 6-8, 10-15, 28-30, and $\geq 42$ days and reduced BUN levels at 2-3, 4-5, 6-8, and $\geq 28$ days. We also found that the MSC group had a lower UAE than the control group. It has been previously shown that MSC treatment reduces the levels of Scr, BUN, and proteinuria in lupus nephritis in mice [38]. Chen et al. [39] found that MSC ameliorates ischemia/reperfusion injuryinduced acute kidney injury in rats and reduces Scr levels. Xiu et al. [40] found that MSC transplantation significantly reduces the concentration of BUN and Scr, prevents tissue injury, and reduces mortality after lipopolysaccharideinduced acute kidney injury. Clinical trials also supported that MSC injection decreases rejection after transplantation. Tan et al. [41] found that the therapy with MSCs achieve better renal function and lower incidence of acute rejection at 1 year compared with the anti-IL-2 receptor antibody induction. Vanikar et al. [42] demonstrated that infusion of MSCs as well as hematopoietic stem cells eases immunosuppression in living donor renal transplantation. Our previous metaanalysis also found that MSCs reduce Scr levels, BUN levels, and proteinuria, as well as alleviate renal damage in animal models of AKI [43]. Lower proteinuria was also found in patients with SLE after MSC therapy [44].

The MSC treatment group had a higher level of GSH, SOD, and a lower level of MDA when compared with the control group. El-Metwaly et al. [45] found that MSCs increase GSH levels and reduce MDA levels in lung tissue of rats subjected to acute lung injury. Li et al. [46] reported that MSCs can restore the levels of GSH and MDA in rats with chronic alcoholism, and its effects on repairing sciatic nerve were obvious. Liu et al. [47] reported that MSCs significantly increase the activity of glutathione (GSH) and 


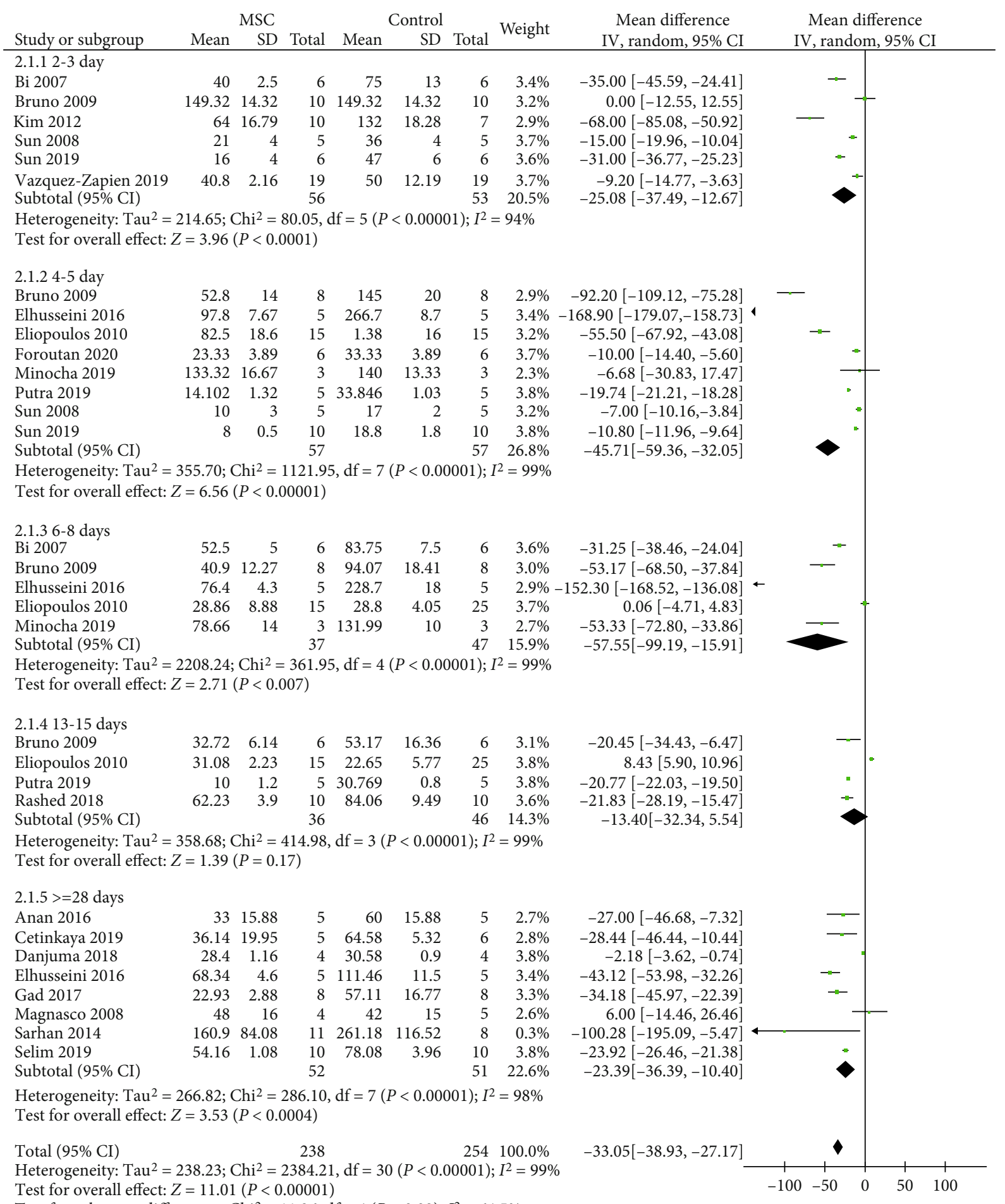

Test for subgroup differences: $\mathrm{Chi}^{2}=11.26, \mathrm{df}=4(P=0.02) ; I^{2}=64.5 \%$

Figure 4: Effect of MSC on BUN.

reduce the levels of MDA in rats induced by unilateral ureteral obstruction.

The mechanism by which MSCs repair injured kidneys may be complex. After kidney injury, VCAM-1, GFP, SDF $-1 /$ CXCR4, and CD44 are upregulated in the injured tissue, which may play important roles in the migration of MSCs to the damaged area. These substances may be partly secreted by the MSCs themselves [20,48, 49]. The presence of MSCs may limit the injury and repair the ischemic tubular damage to maintain the glomerular filtration rate and downregulate BUN [50]. In addition, MSCs lower the expression of several proinflammatory cytokines such as TNF- $\alpha$, IL- $1 \beta$, and IFN- $\gamma$ 


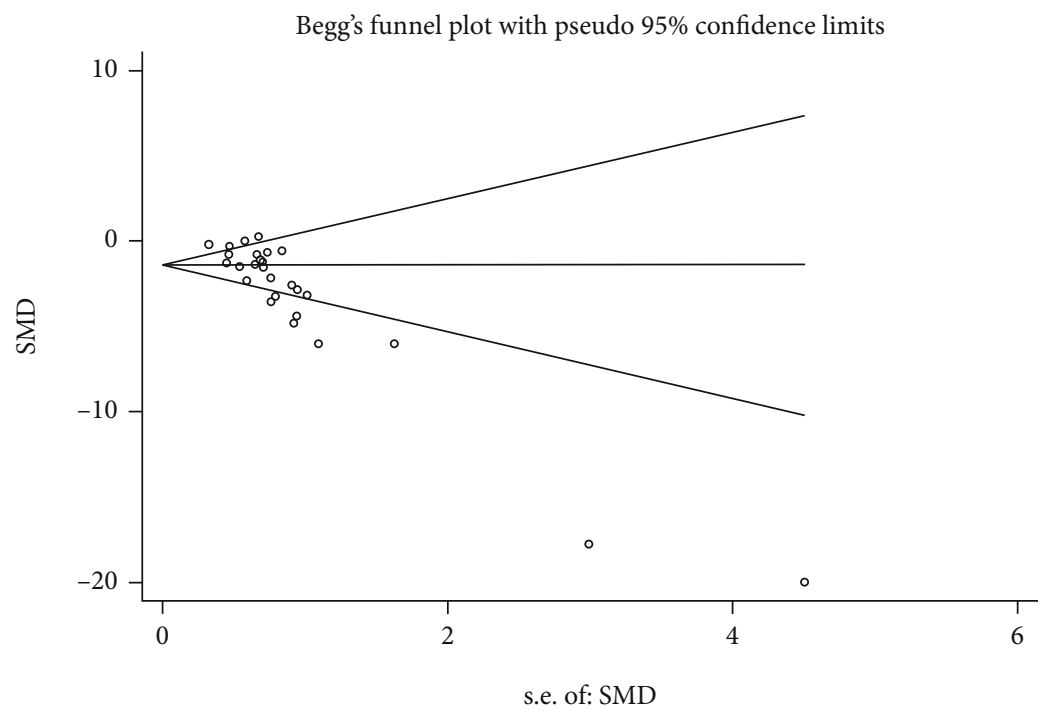

FIGURE 5: Publication bias.

as well as increase anti-inflammatory cytokines such as IL-1, IL-10, Bcl-2, TNF- $\alpha$, bFGF, and prostaglandin E2 $[49,51]$. Another possibility is that MSCs may restore damaged cells and prevent apoptosis by secreting microvesicles, which contain microRNAs, mRNAs, or proteins [49]. To conclude, MSCs can migrate to the damaged tissue, promote the recovery of renal function, enhance proliferation, and reduce fibrosis and inflammation.

Furthermore, our study indicates that MSC treatment can alleviate inflammatory cells, necrotic tubules, regenerative tubules, and renal interstitial fibrosis in kidney disease induced by toxicants. Some previous studies indicated that MSC treatment can alleviate renal pathological changes in unilateral ureteral obstruction rat or mice $[9,10,52]$.

However, this meta-analysis also has some limitations. First, a small sample size was found for the recruited studies. The administered dose and the type of MSCs were not exactly the same. Publication bias was found in this meta-analysis, and the results should be reassessed in the future. Furthermore, the studies frequently had different animal models (mouse or rat), toxin doses, and administration routes for renal injury. These limitations may affect the robustness of our results.

\section{Conclusions}

The MSC treatment reduced Scr levels after 2, 4, 5, 6-8, 10-15, 28-30, and $\geq 42$ days and reduced BUN levels after 2-3, 4-5, 68 , and $\geq 28$ days. The results also indicate that MSC treatment alleviated the inflammatory cells, necrotic tubules, regenerative tubules, and renal interstitial fibrosis in kidney disease induced by toxicants.

\section{Abbreviations}

MSCs: Mesenchymal stem cells

Scr: $\quad$ Serum creatinine

BUN: Blood urea nitrogen
UAE: Urinary albumin excretion

MDA: Malondialdehyde

GSH: L-glutathione

SOD: Superoxide dismutase

WMDs: Weighted mean differences

CI: $\quad$ Confidence intervals

M-H: Mantel-Haenszel.

\section{Data Availability}

The data supporting this meta-analysis are from previously reported studies and datasets, which have been cited. The processed data are available from the corresponding author upon request.

\section{Consent}

There are no human subjects in this article and informed consent is not applicable.

\section{Conflicts of Interest}

The authors declare that they have no competing interests.

\section{Authors' Contributions}

TBZ contributed to the conception and design of the study. TBZ and SJL were responsible for collection of data and performing the statistical analysis and manuscript preparation. WSL and CLL were responsible for checking the data. All authors were responsible for drafting the manuscript, read, and approved the final version.

\section{References}

[1] D. P. Basile, J. V. Bonventre, R. Mehta et al., "Progression after AKI: understanding maladaptive repair processes to predict and identify therapeutic treatments," Journal of the American Society of Nephrology, vol. 27, no. 3, pp. 687-697, 2016. 
[2] T. J. Pianta, N. A. Buckley, P. W. Peake, and Z. H. Endre, "Clinical use of biomarkers for toxicant-induced acute kidney injury," Biomarkers in Medicine, vol. 7, no. 3, pp. 441-456, 2013.

[3] Y. Liang, D. Zhang, L. Li et al., "Exosomal microRNA-144 from bone marrow-derived mesenchymal stem cells inhibits the progression of non-small cell lung cancer by targeting CCNE1 and CCNE2," Stem Cell Research \& Therapy, vol. 11, no. 1, p. $87,2020$.

[4] Jasmin, "In vitro labeling mesenchymal stem cells with superparamagnetic iron oxide nanoparticles: efficacy and cytotoxicity," in Nanoparticles in Biology and Medicine, E. Ferrari and M. Soloviev, Eds., vol. 2118 of Methods in Molecular Biology, pp. 235-250, Humana, New York, NY, 2020.

[5] Y. Zhu, X. Zhang, R. Gu et al., "LAMA2 regulates the fate commitment of mesenchymal stem cells via hedgehog signaling," Stem Cell Research \& Therapy, vol. 11, no. 1, p. 135, 2020.

[6] J. He, Y. L. Jiang, Y. Wang, X. J. Tian, and S. R. Sun, "Microvesicles from mesenchymal stem cells over-expressing miR$34 \mathrm{a}$ inhibit transforming growth factor- $\beta 1$-induced epithelial-mesenchymal transition in renal tubular epithelial cells in vitro," Chinese Medical Journal, vol. 133, no. 7, pp. 800-807, 2020.

[7] D. Li, D. Zhang, B. Tang et al., "Exosomes from human umbilical cord mesenchymal stem cells reduce damage from oxidative stress and the epithelial-mesenchymal transition in renal epithelial cells exposed to oxalate and calcium oxalate monohydrate," Stem Cells International, vol. 2019, Article ID 6935806, 10 pages, 2019.

[8] B. Liu, F. Ding, D. Hu et al., "Human umbilical cord mesenchymal stem cell conditioned medium attenuates renal fibrosis by reducing inflammation and epithelial-to-mesenchymal transition via the TLR4/NF- $\kappa \mathrm{B}$ signaling pathway in vivo and in vitro," Stem Cell Research \& Therapy, vol. 9, no. 1, p. 7, 2018.

[9] L. Xing, E. Song, C. Y. Yu et al., "Bone marrow-derived mesenchymal stem cells attenuate tubulointerstitial injury through multiple mechanisms in UUO model," Journal of Cellular Biochemistry, vol. 120, no. 6, pp. 9737-9746, 2018.

[10] J. Zheng, Q. Wang, W. Leng, X. Sun, and J. Peng, "Bone marrow-derived mesenchymal stem cell-conditioned medium attenuates tubulointerstitial fibrosis by inhibiting monocyte mobilization in an irreversible model of unilateral ureteral obstruction," Molecular Medicine Reports, vol. 17, no. 6, pp. 7701-7707, 2018.

[11] H. H. Anan, R. A. Zidan, M. A. Shaheen, and E. A. Abd- el Fattah, "Therapeutic efficacy of bone marrow derived mesenchymal stromal cells versus losartan on adriamycin-induced renal cortical injury in adult albino rats," Cytotherapy, vol. 18, no. 8, pp. 970-984, 2016.

[12] B. Bi, R. Schmitt, M. Israilova, H. Nishio, and L. G. Cantley, "Stromal cells protect against acute tubular Injuryviaan endocrine effect," Journal of the American Society of Nephrology, vol. 18, no. 9, pp. 2486-2496, 2007.

[13] S. Bruno, C. Grange, M. C. Deregibus et al., "Mesenchymal stem cell-derived microvesicles protect against acute tubular injury," Journal of the American Society of Nephrology, vol. 20, no. 5, pp. 1053-1067, 2009.

[14] B. Cetinkaya, G. Unek, D. Kipmen-Korgun, S. Koksoy, and E. T. Korgun, "Effects of human placental amnion derived mesenchymal stem cells on proliferation and apoptosis mechanisms in chronic kidney disease in the rat," International Journal of Stem Cells, vol. 12, no. 1, pp. 151-161, 2019.
[15] L. Danjuma, P. L. Mok, A. Higuchi et al., "Modulatory and regenerative potential of transplanted bone marrow-derived mesenchymal stem cells on rifampicin-induced kidney toxicity," Regenerative Therapy, vol. 9, pp. 100-110, 2018.

[16] H. A. M. Elbaghdady, M. A. Alwaili, and R. S. El-Demerdash, "Regenerative potential of bone marrow mesenchymal stem cells on cadmium chloride-induced hepato-renal injury and testicular dysfunction in sprague dawley rats," Ecotoxicology and Environmental Safety, vol. 164, pp. 41-49, 2018.

[17] F. M. Elhusseini, M. A. Saad, N. Anber et al., "Long term study of protective mechanisms of human adipose derived mesenchymal stem cells on cisplatin induced kidney injury in Sprague-Daweley rats," Journal of Stem Cells and Regenerative Medicine, vol. 12, no. 1, pp. 36-48, 2016.

[18] N. Eliopoulos, J. Zhao, M. Bouchentouf et al., "Human marrow-derived mesenchymal stromal cells decrease cisplatin renotoxicity in vitro and in vivo and enhance survival of mice post-intraperitoneal injection," American Journal of Physiology-Renal Physiology, vol. 299, no. 6, pp. F1288F1298, 2010.

[19] A. M. Gad, W. A. Hassan, and E. M. Fikry, "Significant curative functions of the mesenchymal stem cells on methotrexate-induced kidney and liver injuries in rats," Journal of Biochemical and Molecular Toxicology, vol. 31, no. 8, 2017.

[20] M. B. Herrera, B. Bussolati, S. Bruno, V. Fonsato, G. M. Romanazzi, and G. Camussi, "Mesenchymal stem cells contribute to the renal repair of acute tubular epithelial injury," International Journal of Molecular Medicine, vol. 14, no. 6, pp. 1035-1041, 2004.

[21] J. H. Kim, D. J. Park, J. C. Yun et al., "Human adipose tissuederived mesenchymal stem cells protect kidneys from cisplatin nephrotoxicity in rats," American Journal of Physiology-Renal Physiology, vol. 302, no. 9, pp. F1141-F1150, 2012.

[22] A. Magnasco, M. Corselli, R. Bertelli et al., "Mesenchymal stem cells protective effect in adriamycin model of nephropathy," Cell Transplantation, vol. 17, no. 10-11, pp. 1157-1167, 2008.

[23] F. E. Moustafa, M. A. Sobh, M. Abouelkheir et al., "Study of the effect of route of administration of mesenchymal stem cells on cisplatin-induced acute kidney injury in Sprague Dawley rats," International Journal of Stem Cells, vol. 9, no. 1, pp. 79-89, 2016.

[24] A. Putra, D. Pertiwi, M. N. Milla et al., "Hypoxia-preconditioned MSCs have superior effect in ameliorating renal function on acute renal failure animal model," Open Access Macedonian Journal of Medical Sciences, vol. 7, no. 3, pp. 305-310, 2019.

[25] H. Qian, H. Yang, W. Xu et al., "Bone marrow mesenchymal stem cells ameliorate rat acute renal failure by differentiation into renal tubular epithelial-like cells," International Journal of Molecular Medicine, vol. 22, no. 3, pp. 325-332, 2008.

[26] L. A. Rashed, S. Elattar, N. Eltablawy, H. Ashour, L. M. Mahmoud, and Y. El-Esawy, "Mesenchymal stem cells pretreated with melatonin ameliorate kidney functions in a rat model of diabetic nephropathy," Biochemistry and Cell Biology, vol. 96, no. 5, pp. 564-571, 2018.

[27] M. Sarhan, H. El Serougy, A. M. Hussein et al., "Impact of bone-marrow-derived mesenchymal stem cells on adriamycin-induced chronic nephropathy," Canadian journal of physiology and pharmacology, vol. 92, no. 9, pp. 733-743, 2014. 
[28] R. E. Selim, H. H. Ahmed, S. H. Abd-Allah et al., "Mesenchymal stem cells: a promising therapeutic tool for acute kidney injury," Applied Biochemistry and Biotechnology, vol. 189, no. 1, pp. 284-304, 2019.

[29] J. H. Sun, G. J. Teng, Z. L. Ma, and S. H. Ju, "In vivo monitoring of magnetically labeled mesenchymal stem cells administered intravascularly in rat acute renal failure," Swiss Medical Weekly, vol. 138, no. 27-28, pp. 404-412, 2008.

[30] M. B. Zickri, S. Zaghloul, M. Farouk, and M. M. Fattah, "Effect of stem cell therapy on adriamycin induced tubulointerstitial injury," International Journal of Stem Cells, vol. 5, no. 2, pp. 130-139, 2012.

[31] M. M. Mata-Miranda, C. E. Bernal-Barquero, A. MartinezCuazitl et al., "Nephroprotective effect of embryonic stem cells reducing lipid peroxidation in kidney injury induced by cisplatin," Oxidative Medicine and Cellular Longevity, vol. 2019, Article ID 5420624, 14 pages, 2019.

[32] G. J. Vazquez-Zapien, A. Martinez-Cuazitl, L. S. Rangel-Cova, A. Camacho-Ibarra, and M. M. Mata-Miranda, "Biochemical and histological effects of embryonic stem cells in a mouse model of renal failure," Romanian journal of morphology and embryology, vol. 60, no. 1, pp. 189-194, 2019.

[33] E. Minocha, R. A. Sinha, M. Jain, C. P. Chaturvedi, and S. Nityanand, "Amniotic fluid stem cells ameliorate cisplatininduced acute renal failure through induction of autophagy and inhibition of apoptosis," Stem Cell Research \& Therapy, vol. 10, no. 1, p. 370, 2019.

[34] B. Sun, X. Luo, C. Yang et al., "Therapeutic effects of human urine-derived stem cells in a rat model of cisplatin-induced acute kidney injury in vivo and in vitro," Stem Cells International, vol. 2019, Article ID 8035076, 13 pages, 2019.

[35] C. Zhang, S. K. George, R. Wu et al., "Reno-protection of urine-derived stem cells in a chronic kidney disease rat model induced by renal ischemia and nephrotoxicity," International Journal of Biological Sciences, vol. 16, no. 3, pp. 435-446, 2020.

[36] W. Sun, Q. Zhu, L. Yan, and F. Shao, "Mesenchymal stem cells alleviate acute kidney injury via miR-107-mediated regulation of ribosomal protein S19," Annals of Translational Medicine, vol. 7, no. 23, p. 765, 2019.

[37] T. Foroutan, M. Nafar, and E. Motamedi, "Intraperitoneal injection of graphene oxide nanoparticle accelerates stem cell therapy effects on acute kidney injury," Stem Cells Cloning, vol. 13, pp. 21-32, 2020.

[38] T. Zhou, C. Liao, H. Y. Li, W. Lin, S. Lin, and H. Zhong, "Efficacy of mesenchymal stem cells in animal models of lupus nephritis: a meta-analysis," Stem Cell Research \& Therapy, vol. 11, no. 1, p. 48, 2020.

[39] Y. Chen, X. Tang, P. Li et al., "Bone marrow derived mesenchymal stromal cells ameliorate ischemia/reperfusion injuryinduced acute kidney injury in rats via secreting tumor necrosis factor-inducible gene 6 protein," BioMed Research International, vol. 2019, Article ID 9845709, 12 pages, 2019.

[40] G.-H. Xiu, X. Zhou, X.-L. Li et al., "Role of bone marrow mesenchymal stromal cells in attenuating inflammatory reaction in lipopolysaccaride-induced acute kidney injury of rats associated with TLR4-NF-kappa B signaling pathway inhibition," Annals of Clinical \& Laboratory Science, vol. 48, no. 6, pp. 743-750, 2018.

[41] J. Tan, W. Wu, X. Xu et al., "Induction therapy with autologous mesenchymal stem cells in living-related kidney transplants: a randomized controlled trial," JAMA, vol. 307, no. 11, pp. 1169-1177, 2012.
[42] A. V. Vanikar, H. L. Trivedi, A. Kumar et al., "Co-infusion of donor adipose tissue-derived mesenchymal and hematopoietic stem cells helps safe minimization of immunosuppression in renal transplantation - single center experience," Renal Failure, vol. 36, no. 9, pp. 1376-1384, 2014.

[43] T. Zhou, C. Liao, S. Lin, W. Lin, H. Zhong, and S. Huang, "The efficacy of mesenchymal stem cells in therapy of acute kidney injury induced by ischemia-reperfusion in animal models," Stem Cells International, vol. 2020, Article ID 1873921, 2020.

[44] T. Zhou, H. Y. Li, C. Liao, W. Lin, and S. Lin, "Clinical efficacy and safety of mesenchymal stem cells for systemic lupus erythematosus," Stem Cells International, vol. 2020, Article ID 6518508, 11 pages, 2020.

[45] S. El-Metwaly, F. F. El-Senduny, R. S. El-Demerdash, and A. F. Abdel-Aziz, "Mesenchymal stem cells alleviate hydrochloric acid-induced lung injury through suppression of inflammation, oxidative stress and apoptosis in comparison to moxifloxacin and sildenafil," Heliyon, vol. 5, no. 12, p. e02710, 2019.

[46] P. Li, Y. Chen, K. Yang, D. Chen, and D. Kong, "Mechanical characteristics of BMSCs-intervened sciatic nerve in chronic alcohol-intoxicated animal model," International Journal of Neuroscience, pp. 1-7, 2020.

[47] B. Liu, F.-X. Ding, Y. Liu et al., "Human umbilical cordderived mesenchymal stem cells conditioned medium attenuate interstitial fibrosis and stimulate the repair of tubular epithelial cells in an irreversible model of unilateral ureteral obstruction," Nephrology, vol. 23, no. 8, pp. 728-736, 2018.

[48] F. E. Togel and C. Westenfelder, "Mesenchymal stem cells: a new therapeutic tool for AKI," Nature reviews. Nephrology, vol. 6, no. 3, pp. 179-183, 2010.

[49] C. J. Barnes, C. T. Distaso, K. M. Spitz, V. A. Verdun, and A. Haramati, "Comparison of stem cell therapies for acute kidney injury," American Journal of Stem Cells, vol. 5, no. 1, pp. 110, 2016.

[50] S. Kale, A. Karihaloo, P. R. Clark, M. Kashgarian, D. S. Krause, and L. G. Cantley, "Bone marrow stem cells contribute to repair of the ischemically injured renal tubule," Journal of Clinical Investigation, vol. 112, no. 1, pp. 42-49, 2003.

[51] F. Togel, Z. Hu, K. Weiss, J. Isaac, C. Lange, and C. Westenfelder, "Administered mesenchymal stem cells protect against ischemic acute renal failure through differentiation-independent mechanisms," American Journal of Physiology-Renal Physiology, vol. 289, no. 1, pp. F31-F42, 2005.

[52] Z. Wang, S. Li, Y. Wang, X. Zhang, L. Chen, and D. Sun, "GDNF enhances the anti-inflammatory effect of human adipose-derived mesenchymal stem cell-based therapy in renal interstitial fibrosis," Stem Cell Research, vol. 41, article 101605, 2019. 